\title{
The feasibility of a Comprehensive Resilience-building psychosocial Intervention (CREST) for people with dementia in the community: protocol for a non-randomised feasibility study
}

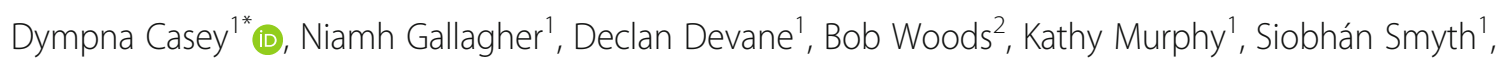
John Newell ${ }^{3}$, Andrew W. Murphy ${ }^{4}$, Charlotte Clarke ${ }^{5}$, Tony Foley ${ }^{6}$, Fergus Timmons ${ }^{7}$, Rose-Marie Dröes ${ }^{8}$, Martin O'Halloran ${ }^{9}$, Gill Windle ${ }^{10}$, Kate Irving Lupton ${ }^{11}$, Christine Domegan ${ }^{12}$, Eamon O'Shea ${ }^{13}$, Pat Dolan ${ }^{14}$ and Priscilla Doyle ${ }^{1}$

\begin{abstract}
Background: A dementia diagnosis can prevent people from participating in society, leading to a further decline in cognitive, social and physical health. However, it may be possible for people with dementia to continue to live meaningful lives and continue to participate actively in society if a supportive psychosocial environment exists. Resilience theory, which focuses on strengthening personal attributes and external assets in the face of serious challenges, may provide a scaffold on which an inclusive multifaceted psychosocial supportive environment can be built. This protocol paper describes a study to determine the feasibility of conducting a multifaceted complex resilience building psychosocial intervention for people with dementia and their caregivers living in the community.
\end{abstract}

Methods: This is a non-randomised feasibility study. Ten participants with dementia and their primary caregivers living in the community will be recruited and receive the CREST intervention. The intervention provides (a) a 7-week cognitive stimulation programme followed by an 8-week physical exercise programme for people with dementia and (b) a 6-week educational programme for caregivers. Members of the wider community will be invited to a dementia awareness programme and GP practices to a dementia training workshop. Trained professionals will deliver all intervention components. Outcomes will assess the feasibility and acceptability of all study processes. The feasibility and acceptability of a range of outcomes to be collected in a future definitive trial, including economic measurements, will also be explored. Finally, social marketing will be used to map a route toward stigma change in dementia for use in a subsequent trial. Quantitative feasibility outcome assessments will be completed at baseline and after completion of the 15-week intervention while qualitative data will be collected at recruitment, baseline, during and post-intervention delivery.

(Continued on next page)

\footnotetext{
* Correspondence: dympna.casey@nuigalway.ie

'School of Nursing \& Midwifery, National University of Ireland Galway, Aras Moyola, Galway, Ireland

Full list of author information is available at the end of the article
}

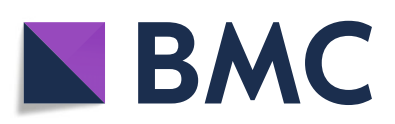

(- The Author(s). 2020 Open Access This article is licensed under a Creative Commons Attribution 4.0 International License, which permits use, sharing, adaptation, distribution and reproduction in any medium or format, as long as you give appropriate credit to the original author(s) and the source, provide a link to the Creative Commons licence, and indicate if changes were made. The images or other third party material in this article are included in the article's Creative Commons licence, unless indicated otherwise in a credit line to the material. If material is not included in the article's Creative Commons licence and your intended use is not permitted by statutory regulation or exceeds the permitted use, you will need to obtain permission directly from the copyright holder. To view a copy of this licence, visit http://creativecommons.org/licenses/by/4.0/ The Creative Commons Public Domain Dedication waiver (http://creativecommons.org/publicdomain/zero/1.0/) applies to the data made available in this article, unless otherwise stated in a credit line to the data. 
(Continued from previous page)

Conclusion: This feasibility study will provide evidence regarding the feasibility and acceptability of a comprehensive multifaceted psychosocial intervention programme for people with dementia and their caregivers (CREST). The results will be used to inform the development and implementation of a subsequent RCT, should the findings support feasibility.

Trial registration: ISRCTN25294519 Retrospectively registered 07.10.2019

Keywords: People with dementia, Caregivers, Resilience, Psychosocial interventions, Non-randomised feasibility study

\section{Background}

Dementia is a progressive neurocognitive disorder [1] characterised by memory and cognitive impairment as well as behaviour changes, all of which impact on the ability of the person with dementia to undertake activities of daily living [2]. It is estimated that there will be 152 million people with dementia worldwide in 2050 [2]. Therefore, dementia is a major contributor to the global burden of disease with a yearly estimated cost of US\$818 billion dollars [3], projected to rise to $\$ 2$ trillion by 2030 [2, 4-6]. Furthermore, almost $85 \%$ of costs are related to family and social care costs [5]. It is not surprising therefore that dementia is considered one of the biggest health, societal and economic challenges of the twentyfirst century [2, 5-7].

A dementia diagnosis may also exacerbate social inequality and discrimination [8]. This diagnosis can lead to marginalisation within the health services [9] and may prevent people with dementia from participating in society $[8,10]$. However, it may be possible for people with dementia to continue to live a meaningful life, retain many abilities and continue to actively participate in society, if there is a supportive psychosocial environment that maximises functioning and social connectedness [5, $11,12]$. Resilience theory is promising as it focuses upon strengthening people's resources in the face of serious challenges and difficulties [13], and may, by enhancing people's strengths and valued relationships, provide a scaffold upon which an inclusive supportive psychosocial environment can be built.

Resilience, defined as a "dynamic and amendable process" [14], encompasses positive adaptation within the context of major adversity. It focuses on modifiable intra-personal skills and protective factors aimed at increasing a person's ability to remain psychologically, socially and physically healthy, or 'resilient', in the face of adversity.

Windle [15] describes resilience as a 'multilevel construct' and a dynamic lifetime process. She presents a resilience framework that focuses on individual, community and societal components that impact the resilience of older people with dementia and their caregivers. Similarly, Harris [16] recommends that resilience-boosting interventions should be multifaceted, targeting the individual, family and community. She outlines that at the individual level, resilience promoting factors include the person with dementia accepting changes in self; an emphasis on nurturing the person's remaining skills; focusing on the positives and what is retained; recognising the multiple ways in which a person with dementia can make a meaningful contribution to family, friends and/or the community. At the level of family and community, resilience-promoting factors include having a supportive doctor; having connections to meaningful community groups and activities, and having a supportive and positive social environment that promotes dignity and respect and reasonable independence. Examining the assets and protective factors both internal and external to the person with dementia is considered central to effective resilience building in people with dementia $[13,14,17,18]$. Many other writers also endorse the position that psychosocial resilience- building interventions need to be multi-faceted, focusing on both the personal attributes and external assets of people with dementia $[15,16,19-22]$.

However, what precisely the preferred components of a multifaceted psychosocial resilience-building intervention might be for people living with dementia is relatively unknown. To address this deficit, we interviewed a sample of people with mild-moderate dementia $(n=6)$ [23], carers $(n=28)$ [24] and 12 informal caregivers/care partners [25]. Analysis of these interviews identified five areas of importance to resilience building: having personal control and a 'fighting spirit', strong family relationships, staying connected to communities, undertaking physical activity and raising awareness and tackling negative attitudes through dementia education.

It is clear from the interviews with people with dementia, caregivers and the literature that psychosocial resilience building interventions need to be multi-faceted, focusing on both the personal attributes and external assets of people with dementia. McDermott et al. [26] carried out a systematic review of the effectiveness of a wide range of psychosocial interventions to meet the physical, cognitive, psychological and social needs of people with dementia. The evidence drawn from 22 reviews evaluating 197 studies indicated that both group cognitive stimulation therapy and physical exercise have recognisable benefits for people with dementia. 
Utilising this evidence and drawing on the expertise of the research team, we identified three successful psychosocial interventions, namely, cognitive stimulation, physical exercise and education, which we hypothesised could be combined to create a novel multifaceted psychosocial resilience-building intervention simultaneously targeting the personal attributes and external assets of people with dementia.

\section{Cognitive stimulation therapy}

Cognitive stimulation therapy (CST) is a nonpharmacological psychosocial intervention aimed at building both internal and external assets of people with mild to moderate dementia [27]. It involves engaging the person with dementia in a range of activities, typically delivered in a group setting, aimed at improving cognitive and social functioning [28]. Many studies reveal that CST has a positive impact on cognitive functioning, and quality of life of people with dementia $[29,30]$ as well as having positive outcomes on social interaction and communication [29]. Furthermore, a Cochrane systematic review on CST, which included 15 randomised controlled trials with a total of 718 participants, found that people with dementia receiving CST had significantly improved cognition, communication and quality of life compared to those receiving usual care or an alternative activity $[26,27]$. CST is also recommended for people with mild to moderate dementia $[10,31]$. CST therefore has the potential to build the internal and external assets of people with dementia.

\section{Exercise}

Physical exercise is important for cognition and brain health because it impacts on other modifiable risks factors such as obesity, hypertension, cardiovascular and metabolic risk factors [5]. Studies also indicate that exercise may decrease inflammation and cholesterol and promote beneficial growth factors in the brain [32, 33]. Although randomised trials have not yet demonstrated that exercise delays cognitive decline or dementia, observational studies do demonstrate an inverse relationship between exercise and risk of dementia [5]. In particular, high-intensity multi-component exercises, including walking, stretching and other strength exercises seemed to be most beneficial [26]. Furthermore, group activities and group exercise interventions with a strong social element have been found to be beneficial and promote social connectedness $[26,34]$ and life satisfaction, and reduce aggression and night-time restlessness $[35,36]$. Likewise, Junge et al. [37] in their systematic mixed studies review exploring the effect and importance of physical activity on behavioural and physiological symptoms of dementia found that of key importance was the 'socially rewarding' aspect of the group-based physical activities.

\section{Dementia education}

Dementia education for informal caregivers, health care professionals and members of the public has been identified as key to enhancing the quality of life of people with dementia [38].

Informal caregivers provide most of the care for people with dementia living at home [39]. These caregivers are therefore a crucial resource/external asset for people with dementia. However, they are often unsupported in their caregiving role, which may result in the person with dementia being placed in residential care prematurely [39]. Dickinson et al. [22] undertook a systematic review of 31 systematic reviews of psychosocial interventions for informal caregivers of people with dementia. They found that effective educational interventions included presenting structured information about dementia and caregiving issues, actively involving caregivers, and delivering the educational programme in a support group format. Other studies reveal that psychosocial educational interventions which include information on how dementia affects the brain, different types of dementia, skills to effectively communicate with people with dementia, how to manage behavioural symptoms, as well as information on local resources have been found to be effective in helping caregivers to more effectively work with, support and care for people with dementia [40-42] and lead to more person- centered attitudes, empathy and improved sense of competence.

Within the community, the general practitioner (GP) is the first health care professional to be consulted when dementia is suspected by the person themselves or by a family member [43]. As outlined by Harris [16], a supportive doctor is an important community level resilience- building resource for people with dementia. However, GPs acknowledge that the complexities associated with the diagnosis and management of dementia is challenging and recognise a need for further education and training [44]. Providing educational support to GP's, in particular interactive small group work [45], may enable them to provide optimal care and promote resilience in people with dementia.

Finally, the broader community and members of the public are key resilience promoting factors for people with dementia, influencing the extent to which the person with dementia can continually function and remain in their own environment [38]. However, a lack of understanding of dementia can lead to fear and stigmatisation, which contributes to the social isolation of people with dementia [46]. Dementia awareness raising interventions can help combat stigma by tackling public perceptions [47]. In particular, public education is 
considered a key strategy in reducing the stigma associated with dementia $[9,46-55]$. The focus is on reducing the knowledge gap [9] and replacing myths with accurate information $[9,49,50,54]$. Social contact in particular is considered the most effective way of reducing stigma and improving attitudes toward dementia [56] as it reduces anxiety about contact and promotes empathy [53]. To be effective, this social contact ideally needs to be targeted, local, credible and sustainable [57], thereby building the external assets of people with dementia. Enhancing and extending the social networks of the person with dementia creates new connections and strengthens relationships [58], thereby building their external resources/assets.

In summary, psychosocial interventions utilising either CST, or physical exercise or education have the capacity to strengthen either personal attributes or external assets of people with dementia. However, multifaceted psychosocial interventions are required to build resilience, which simultaneously target the personal and external assets of people with dementia. We therefore combined CST, exercise and education to create a novel, multifaceted, psychosocial resilience building intervention for use in a future definitive RCT.

\section{Methods}

\section{Study aim}

This study will assess the feasibility of, and inform the optimal design of, a future proposed definitive randomised trial to examine the effectiveness of the CREST intervention for people with dementia and their caregivers living in the community.

\section{Objectives}

1. To assess the feasibility and acceptability of the proposed CREST intervention to participants.

2. To test the feasibility and acceptability of a proposed future definitive trial.

\section{Design}

This study will use a non-randomised feasibility design and will follow the SPIRIT 2013 [59] guidelines and reporting template (Additional file 1). A schedule for enrolment, interventions and assessments is displayed in Fig. 1.

\section{Participants}

\section{People with dementia}

Inclusion criteria

- $>60$ years of age

- Have either i. A formal diagnosis of mild to moderate dementia

ii. Are prescribed dementia medications

iii. Or their GP believes the person has memory problems and the person has a provisional diagnosis of dementia based on the DSM-IV criteria

- And are

- Living in the community

- Willing and capable of undertaking the exercise component of the intervention

- Able to speak and read English

- Able to give informed consent

- Primary care giver is also willing to take part in the study

\section{Caregivers of people with dementia}

Inclusion criteria

- Primary caregiver of a person with dementia who has also agreed to participate in the study

- Does not have a diagnosis of dementia

- Living in the community

- Willing and able to take part in the 6 weeks caregiver education programme

- Able to speak and read English

- Able to give informed consent

\section{Intervention}

The CREST intervention is a multi-level complex psychosocial intervention of 15 weeks duration. It consists of three key interrelated components targeted at people with dementia: their informal caregivers, GPs and the wider community. CREST consists of three components, cognitive stimulation therapy, physical exercise and dementia education. An overview of the intervention components, their duration, content, number and targeted participants is presented in Fig. 2.

\section{1) Cognitive stimulation therapy (CST)}

In this feasibility study, the 'Making a difference' CST programme developed by Spector et al. [12] will be used. This programme consists of 14 sessions delivered over the first 7 weeks of the intervention. In CREST, participants with dementia will attend two $1 \mathrm{~h}$ sessions per week and each session will include approximately five participants. Sessions will include a focus on the use of reminiscence and providing triggers to aid recall, stimulating language and executive functioning and being person-centred.

A health care professional trained to deliver the programme using the CST manual and supported by a co-facilitator will facilitate each session. The role of the 


\begin{tabular}{|c|c|c|c|}
\hline & \multicolumn{3}{|c|}{ STUDY PERIOD } \\
\hline & Enrolment & Post Enrolment & $\begin{array}{l}\text { Close out } \\
(15 \text { weeks) }\end{array}$ \\
\hline TIMEPOINT & $-\mathrm{t} 1$ & t1 & $\mathrm{t} 2$ \\
\hline \multicolumn{4}{|l|}{ ENROLMENT: } \\
\hline Eligibility screen & $\mathrm{x}$ & & \\
\hline Informed consent & $\mathrm{X}$ & & \\
\hline Demographics & $\mathrm{X}$ & & \\
\hline Allocation & & $\mathrm{x}$ & \\
\hline \multicolumn{4}{|l|}{ INTERVENTIONS: } \\
\hline \multicolumn{4}{|l|}{$\begin{array}{l}\text { CST, Physical Exercise, } \\
\text { Dementia Education }\end{array}$} \\
\hline \multicolumn{4}{|l|}{ ASSESSMENTS: } \\
\hline $\begin{array}{l}\text { QoL-AD, MMSE, GDS-15 } \\
\text { SSCQ, AC-QoL, EQ-5D- } \\
\text { 5L, DK-20, SIS, RUD- } \\
\text { Lite, PPOM, ZBI, DAS }\end{array}$ & & $\mathrm{x}$ & $\mathrm{x}$ \\
\hline Interviews & & $\mathrm{X}$ & $X$ \\
\hline
\end{tabular}

Fig. 1 Schedule of enrolment, interventions and assessments as per SPIRIT 2013

co-facilitator will be to help set up the class and support, prompt and encourage participants to adhere to the programme.

\section{2) Physical exercise programme}

An exercise programme, based on the PRINCE structured exercise programme [60] modified to meet the needs of people with dementia, will be used. This exercise programme, delivered over 8 weeks (after the CST programme), is a total of $12 \mathrm{~h}$ in duration: $1 \mathrm{~h}$ session a week for 4 weeks and $2 \mathrm{~h}$ sessions a week for 4 weeks (see Fig. 2). The programme is designed to be delivered in a group class and consists of a combination of aerobic and strength training exercises appropriate for people with dementia and education content specific to exercising. A physical exercise specialist supported by an exercise manual will facilitate the programme. Older adults from the community will support participants with dementia to undertake the exercises. The older adults will be invited to assist in the exercise sessions if they are:

- >60 years of age

- Living in the community

- Do not have a diagnosis of dementia

- Willing and capable of undertaking the exercise programme
- Willing to support people with dementia to undertake the exercise programme

- Able to speak in and read English

- Able to give informed consent

These older adults, who form part of the intervention, will assist people with dementia by prompting them through their exercises and providing support, encouragement as well as social interaction and engagement. They will also assist people with dementia to record and track their exercise progress.

The exercise specialist will assess the progress, duration and intensity of the programme for people with dementia throughout the intervention, in order to increase participants' exercise capacity gradually. In week 1, participants with dementia will be asked to identify specific exercise-related goals they wish to achieve by the end of the programme. These will be reviewed in weeks 4 and 8 . The exercise programme will begin with $10 \times 3$-min circuits. Participants with dementia will start at a station and rotate to the next station until all 10 circuits are completed. Each person with dementia will undertake the exercise at each station 8-10 times with three repetitions. At the end of $3 \mathrm{~min}$, the person will record their exercises and move to the next station. In week 3 , the exercise specialist will assess participants and prescribe weights for strengthening. Participants will 
CREST Intervention: 15 weeks

\begin{tabular}{|c|c|c|c|c|}
\hline \multicolumn{5}{|c|}{ CREST Intervention: 15 weeks } \\
\hline \multirow[b]{2}{*}{ 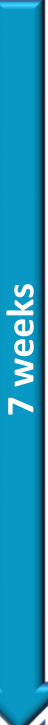 } & $\begin{array}{l}\text { CST: 'Making a Difference' } \\
\text { Programme } \\
\text { (7 weeks) }\end{array}$ & $\begin{array}{c}\text { Dementia Education } \\
\text { Programme for Carers } \\
\text { ( } 6 \text { weeks) }\end{array}$ & $\begin{array}{l}\text { GP Dementia Workshop: } \\
\text { GP PREPARED } \\
\text { (1.5 hours) }\end{array}$ & $\begin{array}{c}\text { Community Dementia } \\
\text { Awareness Programme } \\
\text { (1 hour) }\end{array}$ \\
\hline & $\begin{array}{l}\text { Participants: People with } \\
\text { dementia (n=10) } \\
\text { Content: A group-based } \\
\text { programme consisting of } 14 \\
\text { sessions of themed activities } \\
\text { including the following topics: } \\
\text { - Physical games } \\
\text { - Childhood memories } \\
\text { - Food, current affairs, faces \& } \\
\text { scenes } \\
\text { - Categorizing objects, } \\
\text { orientation, word association } \\
\text { - Number games, word games, } \\
\quad \text { and team games } \\
\text { Duration: } 1 \text { hour delivered twice a } \\
\text { week for } 7 \text { weeks (14 hours in } \\
\text { total) } \\
\text { Delivered by: A CST trained } \\
\text { facilitator and supported by a co- } \\
\text { facilitator. Facilitator manual } \\
\text { provided. } \\
\text { Location: Community }\end{array}$ & $\begin{array}{l}\text { Participants: Caregivers ( } n=10) \\
\text { Content: A group-based } \\
\text { programme consisting of } 6 \text { sessions } \\
\text { including the following topics: } \\
\text { - Understanding Dementia } \\
\text { The Brain \& How Memory } \\
\text { Works } \\
\text { - Communicating with People } \\
\text { Living with Dementia } \\
\text { Responsive Behaviours } \\
\text { - Person Centred Care } \\
\text { Dooking after Yourself } \\
\text { Duration: } 1 \text { hour } 40 \text { mins delivered } \\
\text { once a week for } 6 \text { weeks (10 hours } \\
\text { in total) } \\
\text { Delivered by: A member of the } \\
\text { research team with expertise in } \\
\text { dementia and education. Facilitator } \\
\text { manual provided. } \\
\text { Location: Community }\end{array}$ & 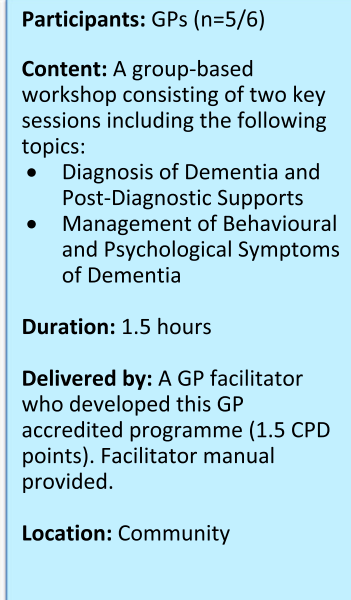 & $\begin{array}{l}\text { Participants: Older adults ( } \mathrm{n}=10 \text { ) } \\
\text { and members of the public } \\
\text { Content: A presentation based on } \\
\text { the HSE's Dementia: Understand } \\
\text { Together Campaign covering the } \\
\text { following topics: } \\
\text { - Dementia prevalence and } \\
\text { impact } \\
\text { - Dementia signs, symptoms and } \\
\text { risk factors } \\
\text { - Stigma and dementia } \\
\text { - Exploring how communities can } \\
\text { support people with dementia } \\
\text { Duration: } 1 \text { hour } \\
\text { Delivered by: A Dementia Elevator } \\
\text { trained facilitator and supported by } \\
\text { a Community Dementia Champion. } \\
\text { Facilitator manual provided. } \\
\text { Location: Community }\end{array}$ \\
\hline & \multicolumn{4}{|c|}{$\begin{array}{l}\text { Physical Exercise Programme } \\
\text { (8 weeks) }\end{array}$} \\
\hline$\underset{\infty}{\infty}$ & \multicolumn{2}{|c|}{$\begin{array}{l}\text { Participants: People with dementia ( } \mathrm{n}=10) \text { \& older adults }(\mathrm{n}=10) \\
\text { Content: A group-based exercise and education programme consisting of } 8 \\
\text { sessions including the following: } \\
\text { - Aerobic and strength training exercises focused on improving } \\
\text { cardiovascular health, strength, balance, coordination and mobility } \\
\text { - Education on the benefits of exercise, behaviour change, motivation, } \\
\text { and strategies for improving brain health }\end{array}$} & \multicolumn{2}{|c|}{$\begin{array}{l}\text { Duration: } 1 \text { hour per week for weeks } 2,5,6,7 \text { and } 2 \text { hours per week for } \\
\text { week } 1,3,4,8 \text {. ( } 12 \text { hours in total) } \\
\text { Delivered by: An exercise specialist. Facilitator manual provided. } \\
\text { Location: Community }\end{array}$} \\
\hline
\end{tabular}

Fig. 2 Overview of the CREST intervention components

be encouraged to complete some of the exercises at home and record these in a home exercise diary. In addition, each person with dementia will be asked to wear a Fitbit wearable activity tracker to measure changes in exercise activity and sleep quality before, during and after the physical exercise programme. Researchers will assist participants to set up their Fitbit and how to access their exercise performance on the tracker. The feasibility and acceptability of wearing this device as well as the extent to which the Fitbit acted as an exercise motivational tool will be captured via qualitative interviews.

\section{3) Dementia education programme}

This programme consists of three elements:

\section{(a) Community dementia awareness programme}

This programme is targeted at members of the public in the wider community. It is designed to provide information on dementia, create positive action for dementia and tackle negative attitudes toward dementia. This programme, based on the Irish Health Service
Executive's [61] Dementia: Understand Together campaign will be $1 \mathrm{~h}$ in duration. It will be delivered and cofacilitated by dementia champions trained to deliver the programme. The programme will include information about dementia prevalence and impact, signs and symptoms, risk factors, and stigma and explores how communities can support the health and well-being of a person with dementia and their family.

\section{(b) Caregiver programme}

This interactive and participatory $10-\mathrm{h}(1 \mathrm{~h} 40 \mathrm{~min}$ a week) educational programme will be delivered over the first 6 weeks (coinciding with the CST programme) (Fig. 2). It will be facilitated by members of the research team with expertise in dementia, nursing and education. This programme is based on the DARES structured education programme [62] modified to meet the needs of informal caregivers of people with dementia and informed by The Alzheimer Society of Ireland's Family Carer Training programme [63]. The aim of this programme is to develop the caregivers' knowledge and skills regarding dementia to enable them to respond more confidently to the needs of the person with dementia, provide them 
with 'me time' and an opportunity to focus on their own health needs and to meet other caregivers and share experiences. An overview of the content of the programme is outlined in Fig. 2.

(c) GP educational workshop

This workshop was developed by the PREPARED team (Primary Care Education, Pathways and Research of Dementia) http://dementiapathways.ie/_filecache/d43/336/634-gpfacilitator-workshop-guide.pdf. This evidence-based 1.5-h interactive, Continuous Professional Development (CPD)accredited dementia workshop aims to support GPs and primary care teams in their delivery of integrated dementia care. Developed following an educational needs analysis, the workshop focuses on dementia diagnosis, post-diagnostic care and management of the behavioural and psychological symptoms of dementia. The peer-facilitated workshop will be delivered by a GP trained to deliver the programme.

\section{Control group}

This will be a single arm feasibility study and will not have a comparison group.

\section{Feasibility study outcomes}

Given the aim of the study, outcomes focus on feasibility and include:

1. Number of participants (people with dementia and caregivers) who are screened, judged eligible and agree to take part in the study.

2. Identification of optimal strategy for recruitment of participants for future definitive trial.

3. Identification of barriers and enablers to stigma change in dementia.

4. Willingness of key gatekeepers, for example, GPs Local Alzheimer Cafés and Western Alzheimer groups, to recruit participants (people with dementia and caregivers).

5. Feasibility and acceptability of the intervention content, delivery and fidelity assessments.

6. Follow-up rates, outcome completion and adherence/compliance rates.

7. Reasons for non-recruitment, non-adherence or attrition.

8. Acceptability of the recruitment process, assessments, data collection tools, intervention content and delivery to participants.

9. Baseline score and variability of secondary outcome measures among participants to inform sample size estimates for a future definitive trial.

10. Evaluation of cost analyses process.

\section{Embedded qualitative study \\ Design}

A qualitative descriptive approach based on the work of Sandelowski [64] will be used to capture key stakeholders views as to the acceptability and feasibility of respective study procedures, including the recruitment process, intervention content and delivery, data collection methods, as well as on the factors that facilitated or hindered engagement and adherence. This data will help identify any feasibility issues, which may need to be addressed to inform the design of a subsequent RCT.

A sample of participants with dementia $(n=5)$ and caregivers $(n=5)$ will be interviewed during the delivery of the CST programme and caregiver programme respectively (weeks 4-5). In addition, a sample of participants with dementia $(n=5)$ will be interviewed during the delivery of the physical exercise programme (weeks 11-12). These interviews will focus on capturing the ongoing perceptions and experiences of participants as they progress through the intervention. All participants will be interviewed post-intervention. Interviewing some participants during and post- intervention will provide information as to how the intervention is progressing and why things may have changed over time as they progressed through the intervention.

The CORTE interviewing framework developed to maximise the meaningful involvement of people with dementia will be used [65]. This guide consists of gaining COnsent, maximizing Responses, Telling the story, and Ending on a positive note. All facilitators/co-facilitators $(n=9)$ as well as the older adults $(n=10)$ supporting the delivery of the exercise component will also be interviewed to capture their experiences of delivering and facilitating the intervention and identify any potential areas for improvement. In total, 54 interviews will be undertaken. Table 1 presents a summary overview of participants who will be interviewed, and the time point for these interviews.

\section{Main trial outcome measurements}

Although this is a feasibility study, we will explore the feasibility and acceptability of a range of secondary outcome measurement tools for use in a future definitive trial. A summary of these measures and the participant from which the outcome will be collected and recorded is presented in Table 2, while Table 3 presents a summary overview of how the pilot may inform a future definitive trial.

\section{Adverse events}

In this study, the definition of an adverse event will be similar to that used in the PRINCE study, i.e. 'Any acute alteration in the patient's physiological condition' [86]. It is not anticipated that participants will be at risk of 
Table 1 Overview of the qualitative data collection process

\begin{tabular}{|c|c|c|c|c|}
\hline Participants & $\begin{array}{l}\text { Number } \\
\text { interviewed } \\
\text { Week 4-5 }\end{array}$ & $\begin{array}{l}\text { Number } \\
\text { interviewed } \\
\text { weeks } 11-12\end{array}$ & $\begin{array}{l}\text { Number interviewed post-intervention } \\
\text { Weeks } 16-19\end{array}$ & $\begin{array}{l}\text { Total number of } \\
\text { interviews }\end{array}$ \\
\hline $\begin{array}{l}\text { People with } \\
\text { dementia }\end{array}$ & 5 (CST) & 5 (Physical Exercise) & 10 & 20 \\
\hline Caregivers & 5 & 0 & 10 & 15 \\
\hline $\begin{array}{l}\text { Facilitators and } \\
\text { co-facilitators }\end{array}$ & & & $\begin{array}{l}4 \text { (CST) } \\
2 \text { (Physical Exercise) } \\
2 \text { (Community Dementia Awareness } \\
\text { programme) } \\
1 \text { (GP Prepared programme) }\end{array}$ & 9 \\
\hline Older adults & & & 10 & 10 \\
\hline Totals & 10 & 5 & 39 & 54 \\
\hline
\end{tabular}

experiencing an adverse event, and participants with dementia will be required to confirm that their GP has been informed and given them permission to participate in the study. Furthermore, the physical exercise programme will be individualised and tailored to each individual participant based on an exercise assessment undertaken by the physical exercise specialist and his/her ongoing weekly monitoring of participants. However, in the unlikely event that an adverse event occurs, this will be recorded by facilitators or members of the research team on an Adverse Event Reporting Form and reported to the participant's GP, as all clinical responsibility rests with those providing routine clinical care.

\section{Health economic analysis}

A preliminary health economic assessment of the intervention will be undertaken. The health care resources consumed will reflect the costs of organising and operating the intervention. These costs will reflect the time input of health professionals and fixed or overhead costs, including any new equipment, training, and capital expenditure related to the intervention. The feasibility and acceptability of collecting data on participants' attendance at their general practice, hospital admissions attendances (both outpatient clinic and emergency room visits) and drug prescriptions as well as any personal expenses incurred in relation to the intervention will also be explored. Economic data will be collected for the month prior to the commencement of the intervention (baseline data) and then during the 15 weeks of the intervention. Exploring the feasibility and acceptability of collecting economic data and the appropriateness of the data collection instruments will inform the design of the economic component of any future trial.

\section{Social marketing}

Social marketing will be used in this study to generate a better understanding of the dynamics at work in relation to stigma and dementia at community level and to map out a route toward stigma change and promote positive action for dementia. Social marketing seeks to develop and integrate marketing concepts with other methods to encourage behaviour change for the benefit of individuals and communities for the greater social good [87]. This process will begin by identifying the barriers and enablers to stigma change from the literature as well as also eliciting the views of people with dementia and caregivers regarding the barriers and enablers for stigma change. The data generated from this analysis will then be categorised and rank ordered by a multidisciplinary core modelling group led by a social marketing expert, to generate a systems map which will model visually any blockages and/or underlying dynamics that affect stigma change in relation to dementia, at a community level. The systems map will also be informed by the quantitative data from the SIS and DAS secondary measurement tools (Table 2). The final stage will involve a focus group interview with local key dementia experts to capture their perspectives and input into the systems map. This will result in the refinement of the map and the identification of leverage points to promote stigma change and help highlight where the focus should be in any subsequent study in relation to promoting stigma change for dementia.

\section{Sample size}

As this is a non-randomised feasibility study, a formal sample size calculation is not required [88]. The aim is to recruit a purposive sample of 10 people with mild to moderate dementia and their primary caregivers $(n=10)$ (20 participants in total), who meet the inclusion criteria, to participate in the CREST intervention. The flow of participants through the study is presented in Fig. 3.

\section{Recruitment}

The initial recruitment plan involved creating a list of GP practices from the HRB Primary Care CTNI within (i) a $30-\mathrm{km}$ radius of the city centre, (ii) having a population of not less than 2500 and (iii) having at least one whole time equivalent practice nurse. These practices were then contacted and invited to respond to an expression of interest regarding participating in the pilot study and to be 
Table 2 Summary overview of secondary outcome measures for future definitive trial

\section{People with dementia: Data collected at baseline and post 15-week intervention}

Quality of Life-Alzheimer's Disease (QoL-AD) [66]. The QoL-AD is a brief, 13-item measure designed specifically to obtain a rating of the person's quality of life from either the person living with dementia and/or the caregiver [66]. For people with dementia, the questionnaire is completed during an interview that usually takes 10-15 min. The QoL-AD has very good psychometric properties and can be completed with people with a wide range of severity of dementia [67]. Internal consistency is good with a Cronbach's alpha coefficient of 0.82 [67]. The scale has good content validity, and it also correlates well with the Dementia Quality of Life scale (0.69) and with the Euroqol-5D scale (0.54), indicating good criterion concurrent validity [67].

Mini-Mental State Examination (MMSE) [68]. The MMSE consists of questions covering 11 domains. It is a simplified, scored form of the cognitive mental status examination, which assesses the cognitive aspects of mental functions.

The Geriatric Depression Scale-Short Form (GDS-15) [69]. This is a 15-item self-completed questionnaire or administered interview used to measure depression in older adults and takes 5-7 min to complete. Mitchell et al. [70] completed a meta-analysis on the diagnostic accuracy, clinical utility and added value of the GDS in primary care. They concluded that the GDS-15 has acceptable sensitivity and specificity (81.3 and $78.4 \%$, respectively), "good" clinical utility for screening and should be used in the diagnosis of late-life depression in primary care.

Stigma Impact Scale (SIS) [71]. The SIS has 24 items and is completed by people with dementia to measure the perceived stigma inherent in progressive neurological diseases. The SIS can be administered via interview or self-completed and on average takes 10 min to complete. The internal consistency has a Cronbach's alpha of 0.89 , indicating good reliability [71]. Construct validity scores range from 0.44 to 0.84 [71].

Positive Psychology Outcome Measure (PPOM) [72]. The PPOM is used to measure hope and resilience in people with dementia. It consists of 16 items designed for either self-completion by participants or completion via an interview and takes 5-10 min to complete. The internal consistency has a Cronbach's alpha of 0.94, indicating excellent reliability [72]. The PPOM remained moderately stable over a 1 week period (ICC: 880), and factor analyses indicated a two-factor structure solution with acceptable fit indices [72]. The PPOM was developed from the Resilience Scale of Wagnild and Young [73], an instrument which scored highly in Windle et al. [74] review as a good measure of resilience.

EQ-5D-5L [75]. The EQ-5D-5L is a 6-item standardized questionnaire that measures self-reported generic health status. The scoring system translates the quality of life scores into an economic value. Domain responses and utility scores have good test-retest reliability (ICC, 0.777 ; agreement, 76.4-98.1\%) [76]. Scores of domains of the EQ-5D-5L correlated significantly $(r, 0.57-0.74)$ with the scores of the SRS-22r domains, supporting construct validity [76].

Fitbit The Fitbit will allow participants' sleep patterns and exercise activity to be monitored. Each Fitbit will be synced to a mobile device or PC. The Fitabase data management platform will be used to extract and aggregate participant data to facilitate analysis.

\section{Caregivers: Data collected at baseline and post 15-week intervention}

Zarit Burden Interview (ZBI) [77]. The ZBI is a 22-item self-completed questionnaire or administered interview used to assess the level of burden experienced by the primary caregivers of older people with dementia and people with disabilities. The ZBI takes on average 10 minutes to complete. It is a valid and reliable instrument with a Cronbach's alpha value of 0.93 ; intra-class correlation coefficient for the test-retest reliability of $0.89(n=149)[78]$

Short Sense of Competence Questionnaire (SSCQ) [79]. The SSCQ is a 7-item self-completed questionnaire or administered interview, which measures the sense of competence the caregiver of a person with dementia has. The SSCQ takes on average 5 min to complete. It is a valid and reliable instrument with a Cronbach's alpha value of 0.76 [79].

Dementia Knowledge 20 (DK-20) [80]. The DK-20 is a 20-item self-administered questionnaire, which measures the knowledge people have regarding dementia. It is a 20-item self-administered questionnaire that takes on average $15 \mathrm{~min}$ to complete. Convergent validity reveals significant correlation between the DK-20 scale and the ADQ calculated using Spearman's one-tailed test, $r(175)=0.36, p<0.001$ [80]. Test-retest reliability for the total scale is ICC $=0.73, p<0.001$, which indicated substantial reliability of the scale [80].

Resource Utilization in Dementia-Lite (RUD-Lite) [81]. The RUD-lite questionnaire consists of 25 items, and measures healthcare resource utilization among older adults with dementia and their caregivers, and time spent on formal and informal care by caregivers. The questionnaire is completed by the primary caregiver of the person with dementia and takes on average 15 minutes to complete. Wimo et al. [82] found the estimations provided by primary caregivers completing the RUD-lite to be accurate. The agreement between diaries and recall estimates was high for personal ADL (intra-class correlation (ICC) 0.93), supervision (ICC 0.87) and total time (ICC 0.91) and lower but acceptable for instrumental ADL (ICC 0.75) [82].

Adult carer quality of life (AC-QoL) [83]. The AC-QoL is a 40-item questionnaire, which measures the overall quality of life of adult caregivers over 8 domains: support for caring, caring choice, caring stress, money matters, personal growth, sense of value, ability to care, and caregiver satisfaction. The questionnaire takes 10 minutes to complete. Construct validity using exploratory factor analysis was conducted by Negri et al. [84] using the Kaiser-Meyer-Olkin (KMO) measure with a total score of 0.90 , and all $\mathrm{KMO}$ values for single items were higher than 0.70 , thus above the acceptability limit of 0.50 [84]. Cronbach alpha scores for the eight subscales range from 0.79 to 0.90 , showing acceptable to excellent levels of reliability; alpha coefficient for the AC-QoL summed score was also excellent (0.93) [84].

\section{Older people: Data collected at baseline and post 15-week intervention}

Dementia Attitudes Scale (DAS) [85]. The dementia attitude's scale is a 20-item questionnaire that measures attitudes towards dementia of members of the public, working professionals, and students. The DAS measures attitudes towards dementia of members of the public, working professionals, and students. DAS correlates significantly with scales that measure ageism and attitudes toward disabilities and has a Cronbach's alphas ranging from 0.83 to 0.85 [85]. 
Table 3 Summary overview of how the pilot may inform a future definitive trial

\begin{tabular}{ll}
\hline $\begin{array}{l}\text { Pilot study-feasibility and acceptabil- } \\
\text { ity of: }\end{array}$ & Informing a future RCT \\
\hline Intervention content \& delivery & Intervention content and modes of delivery finalised \\
Participant recruitment & An optimal participant recruitment strategy identified \\
Training requirements & Research, facilitator and older adult training requirements determined \\
Standard operating procedures (SOPs) & SOPs developed for all study processes \\
Data collection tools (quantitative \& & Quantitative secondary outcome measurement tools, economic data collection tools and qualitative \\
qualitative) & interview guides confirmed \\
Fidelity assessment procedures & Fidelity assessment procedures finalised \\
FITBIT & Feasibility and acceptability of using Fitbit with people with dementia confirmed \\
RCT protocol & RCT protocol developed \\
\hline
\end{tabular}

contacted by a member of the research team. The latter inclusion criteria (ii and iii) were based on the optimal recruitment strategies used in the PRINCE study [60].

GP practices expressing an interest in taking part were asked to contact potential participants with dementia and their primary caregiver (i) informing them of the study, (ii) seeking their consent to participate and (iii) including consent to be contacted by a member of the research team. Participants willing to participate were then to be invited to meet the research team, where consent would be reconfirmed, and participants screened to confirm eligibility and baseline and feasibility data collected using the tools outlined in Table 2. Over a 10-week period, this recruitment strategy yielded only two GP practices out of 14 eligible practices. After another 8-week period, these practices were able to identify 17 participants with dementia who were eligible to participate and consented to be contacted by the research team. Of these, five participants with dementia and their caregivers consented to participate. However, two people with dementia and their caregivers subsequently withdrew, and the remaining six participants (three people with dementia and their caregivers) were retained within the study but an alternative recruitment strategy was then devised as outlined below.

The revised recruitment strategy involves contacting the gatekeepers of local dementia advocacy and health care organisations to identify any potential people with dementia and their caregivers who might be interested in participating in the CREST study. This will include The Alzheimer Society of Ireland, Western Alzheimer's and HSE carer's organisations. The same steps used when recruiting the GP's will be implemented (i) informing potential participants about the study, (ii) seeking their consent to participate and (iii) including consent to be contacted by a member of the research team. Participants willing to participate will then be invited to meet the research team, where consent will be reconfirmed, including permission to contact the participants GP to confirm their eligibility to participate. Baseline and feasibility data will then be collected using the tools outlined in Table 2 .
Older adults $(n=10)$ in the community will be recruited to support the physical exercise element of the intervention using a variety of recruitment methods. These will include, for example, information posters in GP practices, pharmacies, active ageing/retirement groups, older people organisations and support charities, for example, Enable Ireland and St. Vincent de Paul.

Informed consent will be obtained from all participants in the study and process consent, which enables participants to have a collaborative role in the decisions regarding their ongoing participation [89], will be utilised throughout. Older adults, people with dementia and caregivers will all receive $€ 20$ toward travel expenses to facilitate meeting the researchers during recruitment, at each data collection point in the study, as well as when attending each respective session of the intervention.

\section{Criterion for progression to a definitive randomised controlled trial}

Decision-making about progression to a future definitive randomised controlled trial will be based on achieving the criteria for each of the study feasibility outcomes which are outlined in Table 4.

\section{Data management}

The data management process will comply with the General Data Protection Regulation [90] requirements. All participants will be assigned a study number. The master list of participants' names with numeric identifiers will be stored securely away from all other data in a locked filing cabinet with access available only to the members of the research team. All personal data stored on a computer will also be encrypted and passwordprotected in accordance with the General Data Protection Regulation (GDPR) and NUI Galway policies and procedures. Data preparation and cleaning will be conducted prior to data analysis, and any potential identifiers in the qualitative transcripts, e.g. use of names during interview, will be removed. Scores for the questionnaires will be double-checked by two researchers to 


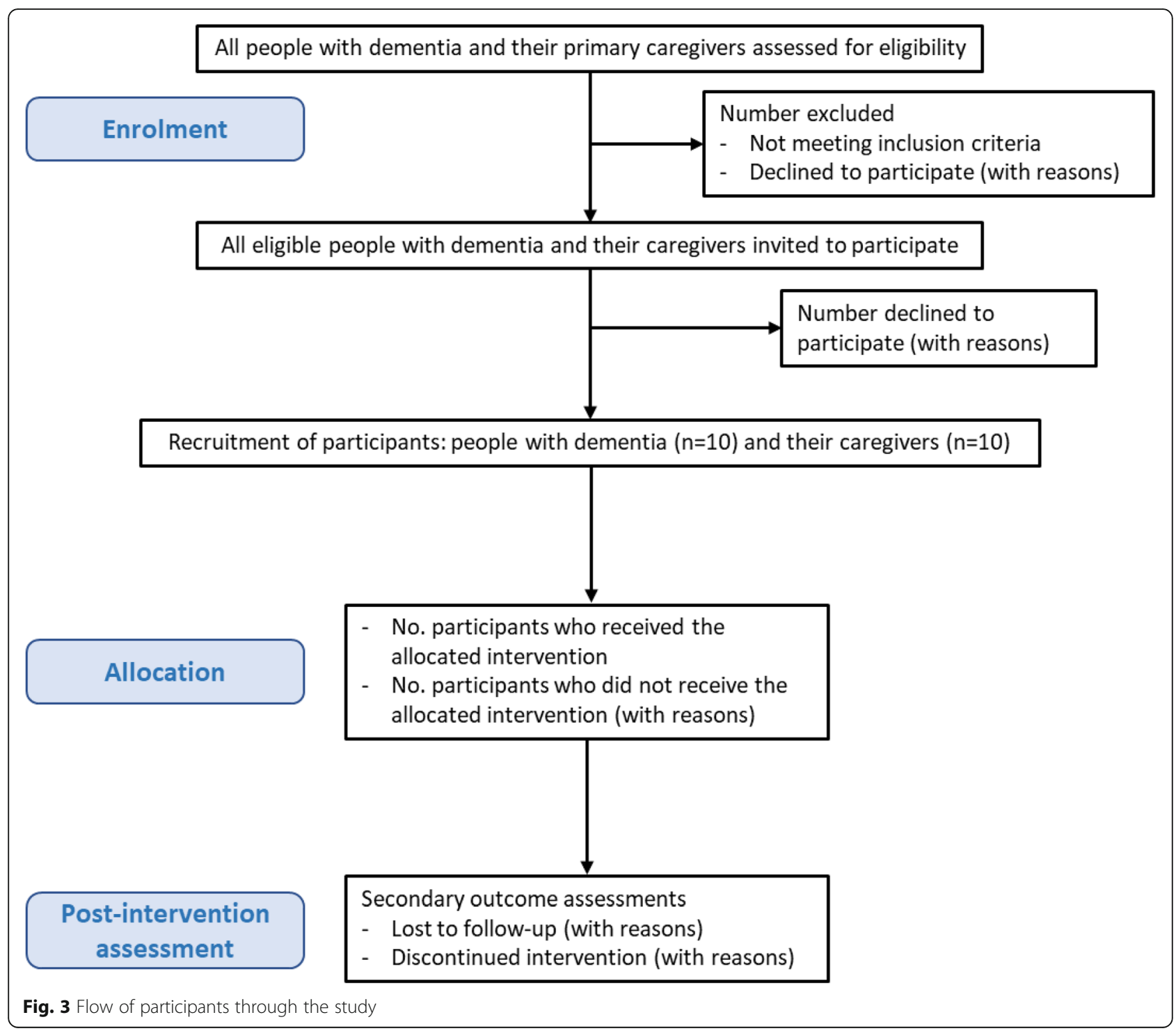

confirm accuracy prior to data entry. Two researchers will also complete a visual record check of the inputted data.

\section{Data analysis}

\section{Quantitative analysis}

All the study questionnaires will be inputted into the SPSS data builder to create a project database. The data can be exported to the software platform (e.g. R (3.5.2) or SPSS (V 26.0) for data cleaning and analysis. Suitable numerical and graphical summaries will be generated for demographic characteristics of the participants (e.g. percentages, measure of central tendency (means or medians), measures of variation (standard deviations or ranges). Statistical analyses appropriate for modelling changes score (e.g. ANCOVA and linear mixed models) will be carried out to estimate changes in the outcome measures from baseline to follow-up. All analyses will adhere to statistical best practice and reproducibility principles.

\section{Qualitative analysis}

Interviews will be recorded and transcribed verbatim, and all participants will be assigned a unique study identifier. Directed qualitative content analysis, based on the work of Hsieh and Shannon [90], will be used to analyse the data, whereby initial coding starts with a theory or relevant research findings. Researchers will then immerse themselves in the data and allow themes to emerge. Initially, each transcript will be read and then open-coded. Each code will be examined and similar codes will be broken down to form categories that are more inclusive. This will lead to a hierarchical structure of categories and subcategories. The final categories will then be arranged into groups ([90], p.1279) that best 


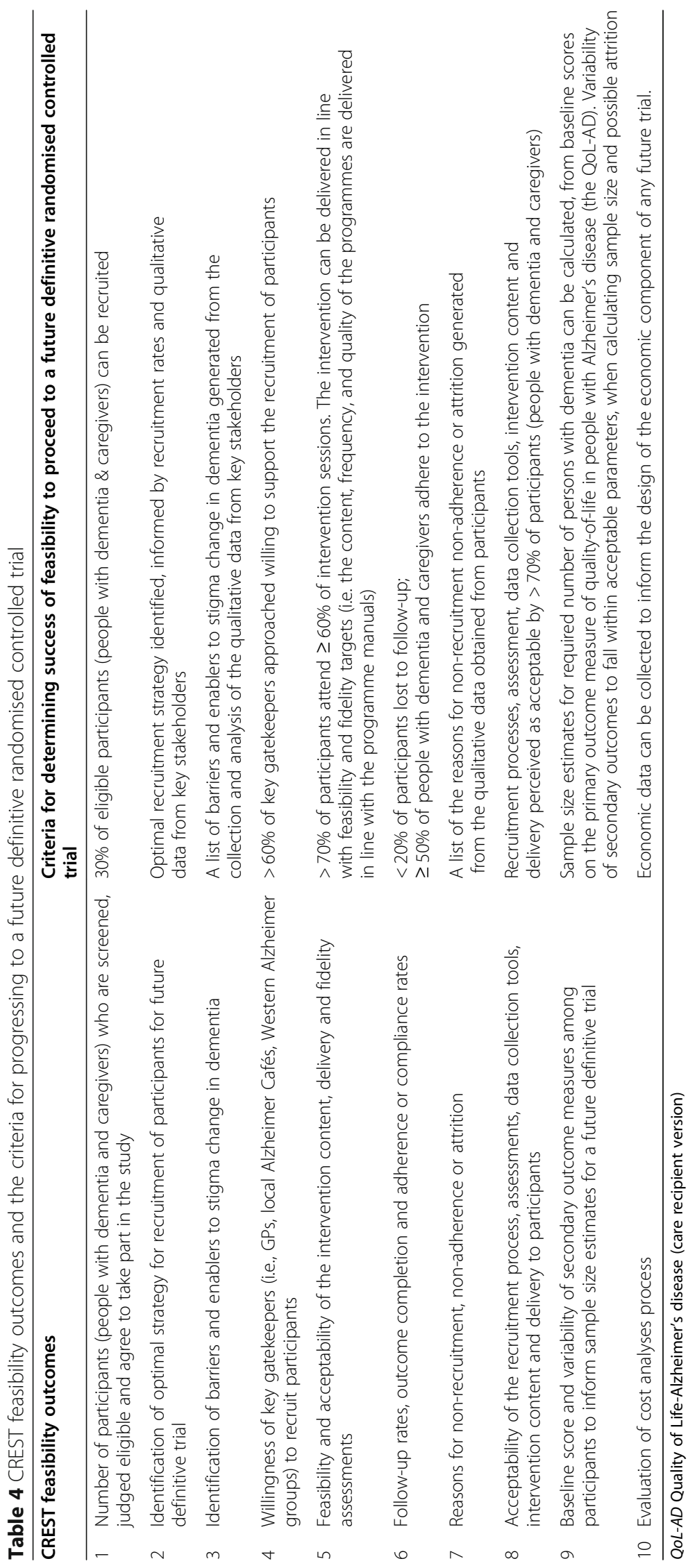


describe the data. The computer software package NVivo (QSR International Pty Ltd., Version 12, 2018) which will be used to facilitate the analysis and strategies to enhance rigour as outlined by Lincoln \& Guba [91] will be put in place. The findings from the qualitative data will be used to improve the intervention and all study procedures and will inform the decision-making in relation to progressing to any future trial.

\section{Fidelity}

Treatment fidelity assessment procedures (quality control of the staff and intervention) will be piloted to provide information on the feasibility of intervention implementation, as well as acceptability and usefulness of treatment fidelity assessment procedures. To maximise treatment fidelity, facilitators/co-facilitators delivering intervention components will be required to complete an adherence to intervention delivery form after the delivery of the components of the intervention. Participants will also be asked to complete an adherence form. An action plan will be drawn up and implemented if a component is not being delivered to the required standard.

\section{Trial management}

A steering group will be established and will consist of key experts including dementia experts, an exercise specialist, psychologists, a trialist, a statistician, a GP, and educationalists. They will provide overall supervision of the trial and help ensure that the trial is conducted rigorously. They will also comment on and approve project documents, research approaches, and findings. In addition, they will review any adverse reports.

\section{Public and patient involvement (PPI)}

Public and patient involvement (PPI) can be described along a spectrum from no involvement (traditional way research has been undertaken) to full partnership. We will establish a Dementia Advisory Forum consisting of people with dementia, caregivers and representatives from various other key stakeholder groups including the Alzheimer Society of Ireland, Western Alzheimers and the HSE carers groups. This advisory forum will be asked for input and advice in reviewing and providing guidance on, for example, study plans, intervention components, content and timing of delivery, data collection methods, interview guides and study results. This will help ensure that the voices and perspectives of targeted end users are heard and are incorporated into the study. PPI involvement will help us to address any issues directly that affect participants, will improve study documents and materials, and will support the dissemination and sharing of achievements in ways that promote accessibility to people with dementia living in the community and their caregivers.

\section{Ethics}

Ethical approval has been obtained from the University Research Ethics committee (Ref: 16-Feb-03; Amend 1907, approval, August $1^{\text {st }}$ 2019). In addition, the university data protection officer has confirmed that the study materials and plans meet the new EU General Data Protection Regulation [92]. We will also comply with simple and clear communication guidelines as recommended by the National Adult Literacy Agency [93] and the HSE [94]. An ethical protocol will also be put in place to ensure that participants' involvement is managed sensitively and without causing upset. In the event of modifications to the protocol, the trial register and the ethics committee will be informed and updates will be made.

\section{Discussion}

Living well with dementia requires the implementation of multifaceted psychosocial resilience- building solutions so that people with dementia can live well for longer within their own communities. This non-randomised feasibility study will determine the feasibility of such an intervention. The results will be disseminated in peerreviewed journals and at national and international conferences and will be used to inform the development and implementation of a subsequent RCT, should the findings support feasibility.

\section{Study status}

Recruitment commenced 21 February 2019 and will end on 14 October 2019.

\section{Supplementary information}

Supplementary information accompanies this paper at https://doi.org/10. 1186/s40814-020-00701-2.

Additional file 1. SPIRIT 2013 Checklist: Recommended items to address in a clinical trial protocol and related documents

\footnotetext{
Abbreviations

ANCOVA: Analysis of covariance; CORTE: Consent, maximizing Responses, Telling the story, and Ending on a positive note; SIS: Stigma Impact Scale; CREST: Comprehensive Resilience-building psychosocial Intervention;

CST: Cognitive stimulation therapy; CTNI: Clinical Trials Network Ireland; DARE S: A dementia education programme incorporating reminiscence therapy for staff; DAS: Dementia Attitudes Scale; DSM-IV: Diagnostic and Statistical Manual of Mental Disorders; GDPR: General Data Protection Regulation; GP: General Practitioner; HRB: Health Research Board; HSE: Health Services Executive; PPI: Public and patient involvement; PREPARED: Primary Care Education, Pathways and Research of Dementia; PRINCE: Pulmonary Rehabilitation Programme for people with Chronic Obstructive Pulmonary Disease in Primary Care; RCT: Randomised controlled trial; SPIRIT: Standard Protocol Items Recommendations for Intervention Trials; SPSS: Statistical Package for Social Sciences
}

Acknowledgements

CREST Dementia Advisory Forum 


\section{Authors' contributions}

All listed authors read and approved the final manuscript. DC, KM, DD and BW conceived and designed the study. JN developed the statistical analysis plan. EOS designed the heath economic component. CD developed the social marketing component. GW, DC, KM, CC, P. Dolan and RMD conceived the theoretical framework. DC, KM, BW, KIL, GW, TF, SS, P. Doyle, NG, MOH and FT contributed to developing the intervention components. AWM, DD, KM, DC, P. Doyle and NG developed the recruitment process. DC created the first draft of the manuscript.

\section{Funding}

The funding for this study was obtained from the Health Research Board following a peer review process. The HRB have no role in the design of the study and collection, analysis and interpretation of data and in writing the manuscript.

\section{Availability of data and materials}

Data sharing is not applicable to this article as no datasets were generated or analysed during the current study.

\section{Ethics approval and consent to participate}

Ethical approval from the National University of Ireland Research Ethics Committee was obtained (Ref: 16-Feb-03); consent to participate was obtained from all participants.

\section{Consent for publication}

Consent for publication is not applicable.

\section{Competing interests}

The authors declare that they have no competing interests.

\section{Author details}

${ }^{1}$ School of Nursing \& Midwifery, National University of Ireland Galway, Aras Moyola, Galway, Ireland. '2Dementia Services Development Centre, Bangor University, Bangor, Wales. ${ }^{3}$ School of Mathematics, Statistics \& Applied Mathematics, National University of Ireland Galway, Galway, Ireland. ${ }^{4}$ Department of General Practice, School of Medicine, National University of Ireland Galway, Galway, Ireland. ${ }^{5}$ Social Sciences and Health, Durham University, Durham, UK. ${ }^{6}$ Department of General Practice, University College Cork, Cork, Ireland. ${ }^{7}$ The Alzheimer Society of Ireland, Temple Road, Blackrock, Co. Dublin, Ireland. ${ }^{8}$ Department of Psychiatry, VU University Medical Center, A.J. Ernststraat 1187 (kamer D0.03), Amsterdam, The Netherlands. ${ }^{9}$ College of Engineering \& Informatics, National University of Ireland Galway, Galway, Ireland. ${ }^{10}$ School of Health Sciences, Bangor University, Bangor, Wales. ${ }^{11}$ School of Nursing, Psychotherapy and Community Health, Dublin City University, Dublin, Ireland. ${ }^{12}$ J.E. Cairnes School of Business \& Economics, National University of Ireland Galway, Galway, Ireland. ${ }^{13}$ Centre for Economic \& Social Research on Dementia, ILAS Building, National University of Ireland, Galway, Ireland. ${ }^{14}$ UNESCO Child and Family Research Centre, School of Political Science and Sociology, National University of Ireland, Galway, Ireland.

Received: 16 October 2019 Accepted: 5 October 2020

Published online: 16 November 2020

\section{References}

1. Woo BK. Dementia health promotion for Chinese Americans. Cureus. 2017; 9(6):e1411.

2. World Health Organisation. Global action plan on the public health response to dementia 2017-2025. Geneva: World Health Organistation; 2017. Available from: https://www.who.int/mental_health/neurology/ dementia/action_plan_2017_2025/en/. Cited 2019 8th October.

3. Prince MJ, Wimo A, Guerchet M, Ali G, Wu Y, Prina M. World Alzheimer Report 2015: the global impact of dementia: an analysis of prevalence, incidence, cost and trends. London: Alzheimer's Disease International; 2015.

4. Frankish $\mathrm{H}$, Horton R. Prevention and management of dementia: a priority for public health. Lancet. 2017;390(10113):e51-e3.

5. Livingston G, Sommerlad A, Orgeta V, Costafreda SG, Huntley J, Ames D, et al. Dementia prevention, intervention, and care. Lancet. 2017;390(10113): 2673.
6. Edick C, Holland N, Ashbourne J, Elliott J, Stolee P. editors. A review of Canadian and international dementia strategies. Healthcare management forum. Los Angeles: SAGE Publications Sage CA; 2017.

7. Carney P, O'Shea E. Philanthropy and dementia care in Ireland. Dementia. 2018;0(0):1-14.

8. Swaffer K. Dementia: stigma, language, and dementia-friendly. London: Sage Publications Sage UK; 2014. p. 706-16.

9. Nolan L, McCarron M, McCallion P, Murphy-Lawless J. Perceptions of stigma in dementia: an exploratory study. Alzheimers Society of Ireland: Dublin; 2006.

10. Batsch NL, Mittelman MS. World Alzheimer Report 2012: overcoming the stigma of dementia. London: Alzheimer's Disease International; 2012.

11. Sabat SR. The experience of Alzheimer's disease: life through a tangled veil. Oxford: Blackhall Publishers Ltd.; 2001.

12. Spector A, Thorgrimsen L, Woods RT, Orrell M. Making a difference: an evidence-based group programme to offer cognitive stimulation therapy (CST) to people with dementia. London: Hawker Publications; 2006.

13. Harris PB. Another wrinkle in the debate about successful aging: the undervalued concept of resilience and the lived experience of dementia. Int J Aging Hum Dev. 2008;67(1):43-61.

14. Luthar SS, Cicchetti D. The construct of resilience: implications for interventions and social policies. Dev Psychopathol. 2000;12(4):857-85.

15. Windle G. The contribution of resilience to healthy ageing. Perspect Public Health. 2012:132(4):159-60.

16. Harris $P$, editor. Is resilience a key to living a meaningful life with dementia? Factors that contribute to the resilience process in early stage dementia. 63rd annual scientific meeting of the Gerontological Society of America. New Orleans: Oxford University Press; 2010.

17. Janssen BM, Van Regenmortel T, Abma TA. Identifying sources of strength: resilience from the perspective of older people receiving long-term community care. Eur J Ageing. 2011;8(3):145-56.

18. Masten AS. Ordinary magic: resilience processes in development. Am Psychol. 2001;56(3):227.

19. Dröes RM, Meiland F, Schmitz M, van Tilburg W. Effect of combined support for people with dementia and carers versus regular day care on behaviour and mood of persons with dementia: results from a multi-centre implementation study. Int J Geriatr Psychiatry. 2004;19(7):673-84.

20. Gaugler JE, Kane RL, Newcomer R. Resilience and transitions from dementia caregiving. J Gerontol B Psychol Sci Soc Sci. 2007;62(1):38-44.

21. Martin-Breen P, Anderies JM. Resilience: a literature review. New York: Rockfeller Foundation; 2011.

22. Dickinson C, Dow J, Gibson G, Hayes L, Robalino S, Robinson L. Psychosocial intervention for carers of people with dementia: what components are most effective and when? A systematic review of systematic reviews. Int Psychogeriatr. 2017;29(1):31-43.

23. Casey D, Murphy K. People with dementia's perceptions of the factors that 927 strengthen resilience. In: 67th annual scientific meeting "makingconnections: 928 from cells to societies" November 2014: The Gerontologist; 2014. p. NP646. https://doi.org/10.13025/S8X590.

24. Murphy K, Casey D. Carers of people with dementia perceptions of resilience and the factors that strengthen resilience. In: 67th annual scientific meeting "making connections: from cells to societies" November 2014. Washington DC: The Gerontologist; 2014. p. NP646.

25. Clarke CL, Bailey C. Narrative citizenship, resilience and inclusion with dementia: on the inside or on the outside of physical and social places. Dementia. 2016;15(3):434-52.

26. McDermott O, Charlesworth G, Hogervorst E, Stoner C, Moniz-Cook E, Spector A, et al. Psychosocial interventions for people with dementia: a synthesis of systematic reviews. Aging Ment Health. 2019;23(4):393-403.

27. Woods B, Aguirre E, Spector AE, Orrell M. Cognitive stimulation to improve cognitive functioning in people with dementia. Cochrane Database Syst Rev. 2012;2 https://doi.org/10.1002/14651858.CD005562.pub2.

28. Clare L, Woods RT. Cognitive training and cognitive rehabilitation for people with early-stage Alzheimer's disease: a review. Neuropsychol Rehabil. 2004; 14(4):385-401.

29. Spector A, Thorgrimsen L, Woods B, Royan L, Davies S, Butterworth M, et al. Efficacy of an evidence-based cognitive stimulation therapy programme for people with dementia: randomised controlled trial. Br J Psychiatry. 2003; 183(3):248-54

30. Aguirre E, Hoare Z, Streater A, Spector A, Woods B, Hoe J, et al. Cognitive stimulation therapy (CST) for people with dementia-who benefits most? Int J Geriatr Psychiatry. 2012;28(3):284-90. 
31. National Institute for Health and Care Excellence (NICE). Dementia: supporting people with dementia and their carers in health and social care. NICE clinical guideline 42, 2006. Available from: www.nice.org.uk/guidance/ cg42. Cited 2019 6th October.

32. Forbes D, Forbes SC, Blake CM, Thiessen EJ, Forbes S. Exercise programs for people with dementia. Cochrane Database Syst Rev. 2015;4 https://doi.org/ 10.1002/14651858.CD006489.pub3.

33. Jensen CS, Hasselbalch SG, Waldemar G, Simonsen AH. Biochemical markers of physical exercise on mild cognitive impairment and dementia: systematic review and perspectives. Front Neurol. 2015;6:187.

34. Hopman-Rock M, Staats PG, Tak EC, Dröes RM. The effects of a psychomotor activation programme for use in groups of cognitively impaired people in homes for the elderly. Int J Geriatr Psychiatry. 1999;14(8):633-42.

35. Dröes RM, Tilburg WV. Effects of psychomotor therapy in patients with senile dementia of the Alzheimer type. In: Colon EJ, editor. The Akon Series "Ageing in the contemporary society"; 1993. p. 23.

36. Dröes R, Van Tilburg W. Amelioration du comportement agressif par des activités psychomotrices [Improvement of agressive behaviour by psychomotor activities]. L'Année Gérontologique. 1996;10:471-82.

37. Junge T, Ahler J, Knudsen HK, Kristensen HK. The effect and importance of physical activity on behavioural and psychological symptoms in people with dementia: a systematic mixed studies review. Dementia. 2018; https:// doi.org/10.1177/1471301218777444.

38. Irving K, Piasek P, Kilcullen S, Coen A-M, Manning M. National educational needs analysis report. Dublin: Dublin City Univeristy and Heatlhcare Service Executive; 2014

39. Cahill S, O'Shea E, Pierce M. Creating excellence in dementia care: a research review for Ireland's national dementia strategy: Dementia Services Information and Development Centre; 2012. http://hdl.handle.net/10147/3 06721.

40. Belle SH, Burgio L, Burns R, Coon D, Czaja SJ, Gallagher-Thompson D, et al. Enhancing the quality of life of dementia caregivers from different ethnic or racial groups: a randomized, controlled trial. Ann Intern Med. 2006;145(10): 727-38.

41. Gitlin L, Hodgson N. Caregivers as therapeutic agents in dementia care: the evidence-base for interventions supporting their role. In: Family caregiving in the new normal. Philadelphia: Elsevier; 2015. p. 305-56.

42. Hattink B, Meiland F, van der Roest H, Kevern P, Abiuso F, Bengtsson J, et al. Web-based STAR E-learning course increases empathy and understanding in dementia caregivers: results from a randomized controlled trial in the Netherlands and the United Kingdom. J Med Internet Res. 2015;17(10):e241.

43. Foley T, Swanwick G. Dementia: diagnosis and management in general practice. Irish College of General Practitioners Quality in Practice Committee: Dublin; 2014.

44. Foley T, Boyle S, Jennings A, Smithson WH. "We're certainly not in our comfort zone": a qualitative study of GPs' dementia-care educational needs. BMC Fam Pract. 2017;18(1):66

45. Koch T, lliffe S. Dementia diagnosis and management: a narrative review of changing practice. Br J Gen Pract. 2011;61(589):e513-e25.

46. World Health Organization. Dementia: a public health priority: World Health Organization; 2012. Available from: https://www.who.int/mental_health/ publications/dementia_report_2012/en/. Cited 2019 8th October.

47. Mukadam N, Livingston G. Reducing the stigma associated with dementia: approaches and goals. Aging Health. 2012;8(4):377-86.

48. Chan JY, Mak WW, Law LS. Combining education and video-based contact to reduce stigma of mental illness: "the same or not the same" anti-stigma program for secondary schools in Hong Kong. Soc Sci Med. 2009;68(8):1521-6.

49. Corrigan PW. Lessons learned from unintended consequences about erasing the stigma of mental illness. World Psychiatry. 2016;15(1):67-73.

50. Corrigan PW. Resolving mental illness stigma: should we seek normalcy or solidarity? Br J Psychiatry. 2016;208(4):314-5.

51. Corrigan PW, Shapiro JR. Measuring the impact of programs that challenge the public stigma of mental illness. Clin Psychol Rev. 2010;30(8):907-22.

52. Corrigan PW, Fong MW. Competing perspectives on erasing the stigma of illness: what says the dodo bird? Soc Sci Med. 2014;103:110-7.

53. Gronholm PC, Henderson C, Deb T, Thornicroft G. Interventions to reduce discrimination and stigma: the state of the art. Soc Psychiatry Psychiatr Epidemiol. 2017;52(3):249-58

54. Rüsch N, Angermeyer MC, Corrigan PW. Mental illness stigma: concepts, consequences, and initiatives to reduce stigma. Eur Psychiatry. 2005;20(8): 529-39.
55. Thomas S, Bestman A, Pitt H, David J, Thomas S. Lessons for the development of initiatives to tackle the stigma associated with problem gambling. Melbourne: Victorian Responsible Gambling Foundation; 2016.

56. Thornicroft G, Mehta N, Clement S, Evans-Lacko S, Doherty M, Rose D, et al. Evidence for effective interventions to reduce mental-health-related stigma and discrimination. Lancet. 2016;387(10023):1123-32.

57. Corrigan PW. Best practices: strategic stigma change (SSC): Five principles for social marketing campaigns to reduce stigma. Psychiatr Serv. 2011;62(8): 824-6.

58. Lakey L, Chandaria K, Quince C, Kane M, Saunders T. Dementia 2012: a national challenge, vol. 2012. London: Alzheimer's Society. p. 68-73.

59. Chan A-W, Tetzlaff JM, Altman DG, Laupacis A, Gøtzsche PC, Krleža-Jerić K, et al. SPIRIT 2013 statement: defining standard protocol items for clinical trials. Ann Intern Med. 2013;158(3):200-7.

60. Casey D, Murphy K, Devane D, Cooney A, McCarthy B, Mee L, et al. The effectiveness of a structured education pulmonary rehabilitation programme for improving the health status of people with moderate and severe chronic obstructive pulmonary disease in primary care: the PRINCE cluster randomised trial. Thorax. 2013:68(10):922-8.

61. Health Service Executive. Dementia: understand together. 2010.

62. O'Shea E, Devane D, Murphy K, Cooney A, Casey D, Jordan F, et al. Effectiveness of a structured education reminiscence-based programme for staff on the quality of life of residents with dementia in long-stay units: a study protocol for a cluster randomised trial. Trials. 2011;12(1):41.

63. The Alzheimers Society of Ireland. Family carer training: The Alzheimers Society of Ireland; 2019. [unpublished training resource]. Available from: The Alzheimers Society of Ireland.

64. Sandelowski M. Whatever happened to qualitative description? Res Nurs Health. 2000;23(4):334-40.

65. Murphy K, Jordan F, Hunter A, Cooney A, Casey D. Articulating the strategies for maximising the inclusion of people with dementia in qualitative research studies. Dementia. 2015;14(6):800-24.

66. Logsdon RG, Gibbons LE, McCurry SM, Teri L. Quality of life in Alzheimer's disease: patient and caregiver reports. J Ment Health Aging. 1999;5:21-32.

67. Thorgrimsen L, Selwood A, Spector A, Royan L, de Madariaga LM, Woods RT, et al. Whose quality of life is it anyway?: the validity and reliability of the quality of life-Alzheimer's disease (QoL-AD) scale. Alzheimer Dis Assoc Disord. 2003;17(4):201-8.

68. Folstein MF, Folstein SE, McHugh PR. "Mini-mental state": a practical method for grading the cognitive state of patients for the clinician. J Psychiatr Res. 1975;12(3):189-98

69. Yesavage JA, Sheikh Jl. Geriatric depression scale (GDS) recent evidence and development of a shorter version. Clin Gerontol. 1986;5(1-2):165-73.

70. Mitchell AJ, Bird V, Rizzo M, Meader N. Diagnostic validity and added value of the Geriatric Depression Scale for depression in primary care: a metaanalysis of GDS30 and GDS15. J Affect Disord. 2010;125(1-3):10-7.

71. Burgener SC, Berger B. Measuring perceived stigma in persons with progressive neurological disease: Alzheimer's dementia and Parkinson's disease. Dementia. 2008;7(1):31-53.

72. Stoner CR, Orrell M, Spector A. The Positive Psychology Outcome Measure (PPOM) for people with dementia: psychometric properties and factor structure. Arch Gerontol Geriatr. 2018;76:182-7.

73. Wagnild GM, Young HM. Development and psychometric evaluation of the Resilience Scale. J Nurs Meas. 1993;1(2):165-7847.

74. Windle G, Bennett KM, Noyes J. A methodological review of resilience measurement scales. Health Qual Life Outcomes. 2011;9(1):8.

75. Herdman M, Gudex C, Lloyd A, Janssen MF, Kind P, Parkin D, Bonsel G, et al. Development and preliminary testing of the new five-level version of EQ-5D (EQ-5D-5L). Qual Life Res. 2011;20(10):1727-36.

76. Cheung PW, Wong CK, Samartzis D, Luk KD, Lam CL, Cheung KM, et al. Psychometric validation of the EuroQoL 5-Dimension 5-Level (EQ-5D-5L) in Chinese patients with adolescent idiopathic scoliosis. Scoliosis Spinal Disord. 2016;11(1):19.

77. Zarit SH, Reever KE, Bach-Peterson J. Relatives of the impaired elderly: correlates of feelings of burden. Gerontologist. 1980;20(6):649-55.

78. Seng BK, Luo N, Ng WY, Lim J, Chionh HL, Goh J, et al. Validity and reliability of the Zarit Burden Interview in assessing caregiving burden. Ann Acad Med Singap. 2010;39:758-63.

79. Vernooij-Dassen MJ, Felling AJ, Brummelkamp E, Dauzenberg MG, van den Bos GA, Grol R. Assessment of caregiver's competence in dealing with the burden of caregiving for a dementia patient: a Short Sense of Competence 
Questionnaire (SSCQ) suitable for clinical practice. J Am Geriatr Soc. 1999; 47(2):256-7.

80. Shanahan N, Orrell M, Schepers AK, Spector A. The development and evaluation of the DK-20: a knowledge of dementia measure. Int Psychogeriatr. 2013;25(11):1899-907.

81. Wimo A, Winblad B. Resource Utilization in Dementia: RUD Lite. Brain Aging 2003;3(1):48-59.

82. Wimo A, Jonsson L, Zbrozek A. The Resource Utilization in Dementia (RUD) instrument is valid for assessing informal care time in community-living patients with dementia. J Nutr Health Aging. 2010;14(8):685-90.

83. Elwick H, Joseph S, Becker S, Becker F. Manual for the adult carer quality of life questionnaire (AC-QoL). London: The Princess Royal Trust for Carers; 2010.

84. Negri L, Piazza G, Sartori RD, Cocchi MG, Delle FA. The adult carer quality of life questionnaire (AC-QoL): comparison with measures of burden and wellbeing, and Italian validation. Disabil Rehabil. 2019;41(10):1207-16.

85. O'Connor ML, McFadden SH. Development and psychometric validation of the Dementia Attitudes Scale. Int J Alzheimers Dis. 2010;454218:10 https:// doi.org/10.4061/2010/454218.

86. Murphy K, Casey D, Devane D, Cooney A, McCarthy B, Mee L, et al. A cluster randomised controlled trial evaluating the effectiveness of a structured pulmonary rehabilitation education programme for improving the health status of people with chronic obstructive pulmonary disease (COPD): the PRINCE Study Protocol. BMC Pulm Med. 2011;11(1):4.

87. Tapp A, Brophy R, Carausan M, Carruthers J, Peattie S, Revill S, et al. The iSMA, ESMA and AASM consensus definition of social marketing: Monash University Library; 2013. Available from: https://www.i-socialmarketing.org/ assets/social_marketing_definition.pdf. Cited 2019 7th October.

88. Billingham SA, Whitehead AL, Julious SA. An audit of sample sizes for pilot and feasibility trials being undertaken in the United Kingdom registered in the United Kingdom Clinical Research Network database. BMC Med Res Methodol. 2013;13(1):104

89. Jokinen P, Lappalainen M, Meriläinen P, Pelkonen M. Ethical issues in ethnographic nursing research with children and elderly people. Scand J Caring Sci. 2002;16(2):165-70.

90. Hsieh H-F, Shannon SE. Three approaches to qualitative content analysis. Qual Health Res. 2005;15(9):1277-88.

91. Lincoln YS, Guba EG. Naturalistic inquiry. Newberry Park: Sage Publications; 1985.

92. EU General Data Protection Regulation: Official Journal of the European Union; 2018. Available from: https:/eur-lex.europa.eu/legalcontent/EN/TXT/ Q151131PDF/?uri=CELEX:32016R0679\&from=EN. Cited 2019 5th October.

93. National Adult Literacy Agency. Writing and design tips. 3rd ed. NALA: Dublin; 2011.

94. Health Service Executive. Guidelines for communicating clearly using plain English with our patients and service users. Dublin: Dr Steevens' Hospital; 2017

\section{Publisher's Note}

Springer Nature remains neutral with regard to jurisdictional claims in published maps and institutional affiliations.

Ready to submit your research? Choose BMC and benefit from:

- fast, convenient online submission

- thorough peer review by experienced researchers in your field

- rapid publication on acceptance

- support for research data, including large and complex data types

- gold Open Access which fosters wider collaboration and increased citations

- maximum visibility for your research: over $100 \mathrm{M}$ website views per year

At BMC, research is always in progress.

Learn more biomedcentral.com/submissions 